\title{
Méthode d'aide à l'idéalisation de modèles issus de la CAO pour le calcul de structures
}

\author{
Yassine Benhafid ${ }^{a}$, Nadège Troussier, Nassim Boudaoud et Zohra Cherfi \\ Laboratoire ODIC. Centre de recherches Pierre Guillaumat, BP 60319, 60203 Compiègne Cedex, France
}

Reçu le 25 février 2005, accepté le 21 mars 2005

\begin{abstract}
Résumé - Le calcul de structures mécaniques est un outil d'aide à la conception aujourd'hui largement utilisé. Quelle que soit la méthode de calcul de structures utilisée, l'adaptation du modèle de conception (géométrie, données technologiques, spécifications fonctionnelles) en un modèle de comportement spécifique au calcul de structures est une étape nécessaire nommée idéalisation. Cette étape particulièrement critique repose principalement sur l'expertise de l'analyste. Cet article propose une méthode d'aide aux choix d'idéalisation d'un modèle CAO en un modèle de comportement. La méthode proposée peut aider un analyste à effectuer ses choix d'idéalisation en respectant la qualité, le coût et les délais de simulation du système analysé. Elle est basée sur l'exploitation de résultats obtenus sur des calculs de référence, jugés similaires au calcul à traiter.
\end{abstract}

Mots clés : Simulation / calcul / CAO / idéalisation / conception

Abstract - An assistance method for idealization of CAD models in structural analysis models. Nowadays, the structural analysis is widely used in mechanical design. Whatever the structural analysis method used, the transformation of the CAD model (geometry, technological parameters, specifications...) in an analysis model for structural analysis is necessary and is called "idealization". This step is especially difficult and requires an expert analyst. The proposed methodology aims at supporting the idealization choices in order to reach cost, quality and time leading specifications. It is based on the use of results obtained on reference cases, judged similar to the calculation case to be driven.

Key words: Simulation / structural analysis / CAD / idealization / design

\section{Introduction}

L'utilisation de la CAO en simulation a des avantages comme des inconvénients. Les avantages de la $\mathrm{CAO}$ se résument dans la capacité de représenter des systèmes physiques virtuellement en utilisant des outils de conception. La CAO utilise généralement des modèles présentant des idéalisations sur le comportement (idéalisations géométriques, des conditions aux limites, etc.). Ces idéalisations sont alors la source d'erreurs sur le comportement simulé par rapport au comportement réel. Il est donc nécessaire de maîtriser l'ensemble du processus de calcul et de maîtriser l'impact des erreurs effectuées au cours des choix d'idéalisation ou de modélisation en calcul de façon à pouvoir interpréter les résultats. Szabo [1] identifie trois types d'erreurs réalisés tout au long d'un processus de calcul : l'erreur théorique, l'erreur de modélisation (ou d'idéalisation) et l'erreur de discrétisation présentées à la figure 1 .

\footnotetext{
a Auteur correspondant : yassine.benhafid@utc.fr
}

L'erreur théorique est due à l'incompréhension ou à la supposition incomplète d'un comportement réel simulé par un analyste. L'erreur de modélisation est le résultat des simplifications conceptuelles, ou idéalisations, apportées dans la formulation mathématique d'un système analysé. L'erreur de discrétisation est due aux simplifications appliquées par une méthode de calcul ou de convergence (comme la méthode des éléments finis par exemple).

Par ailleurs, Troussier a proposé une structuration des modèles pour le calcul basée sur l'identification d'étapes et de liens temporels et de dépendance [2]. Ainsi, selon Troussier, un calcul de structures est composé de six étapes qui sont les suivantes [2] :

Étape 1 : un modèle de conception représente le produit conçu avec les exigences techniques.

Étape 2 : un but de simulation contient l'objectif de l'analyse et définit le niveau de qualité demandée avec le coût maximal et le temps estimé pour le processus de calcul. 


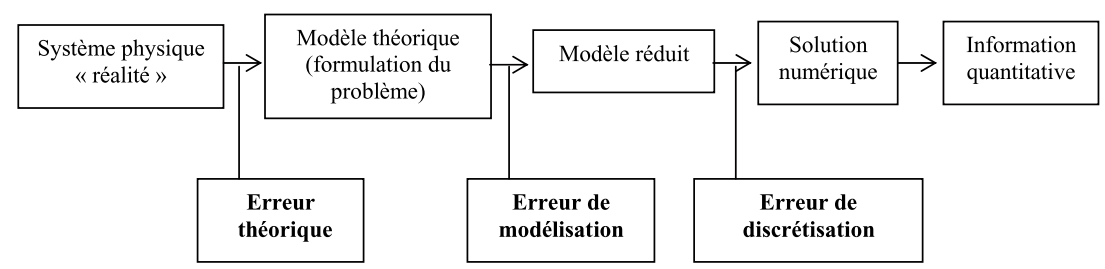

Fig. 1. Les trois types d'erreur à maîtriser lors d'un processus de calcul [1].

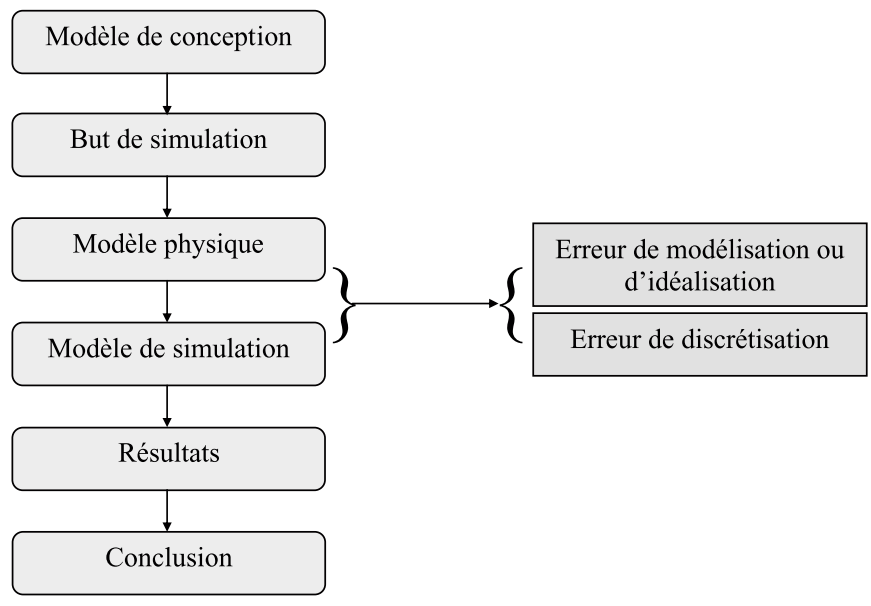

Fig. 2. Les étapes du processus de calcul et les erreurs associées $[2,3]$.

Étape 3 : un modèle physique fournit les hypothèses choisies pour satisfaire le but de simulation. Cette étape est décisive dans le travail de l'analyse mécanique. Généralement, elle est réalisée sur la base de l'expérience de l'analyste.

Étape 4 : un modèle de simulation est construit suite au choix d'une méthode et d'un outil de calcul pour calculer le modèle mécanique. En d'autres termes, si le modèle physique est ce que l'on veut faire, le modèle de simulation est ce que l'on peut faire.

Étape 5: un résultat consiste en un ensemble de résultats fournis par l'outil de calcul.

Étape 6 : une conclusion est donnée à la fin du procédé de l'analyse par un test de validité du processus de calcul. Si le processus est non validé, d'autres calculs seront réalisés. Si le processus est validé, l'analyse des résultats est effectuée pour satisfaire le but de simulation.

La structuration proposée par Troussier aide à la maîtrise d'un calcul de structure par la maîtrise des étapes qui le constituent. Les étapes 4 et 5 sont la source des trois types d'erreur cités précédemment [4]. Si de nombreux travaux existent et ont donné naissance à des outils permettant de mieux maîtriser l'erreur numérique faite au niveau du modèle de simulation, la construction du modèle physique (et par conséquent la maîtrise des erreurs d'idéalisation) reste une tâche experte, délicate et très peu structurée $([5-7] \ldots)$. Comme le dit Pourroy dans [8], « toute la difficulté de la modélisation réside dans le choix des niveaux de simplification les plus judicieux, c'est-à-dire les mieux adaptés :

- à la nature de la grandeur physique à calculer : les modèles mis en œuvre pour le calcul de grandeurs locales (contraintes, déformation ... ) sont généralement plus fins que ceux concernant des grandeurs plus globales (déplacements, fréquences propres ... ) ;

- aux impératifs de délai (cela concerne non seulement les temps de calcul, mais aussi les temps d'élaboration du modèle et d'exploitation des résultats);

- aux impératifs de précision sur les résultats;

- aux impératifs logistiques (disponibilité du personnel, de machines et d'outils appropriés) ».

Notre travail propose d'apporter des éléments de structuration du modèle physique, par une méthode d'aide à l'idéalisation pour un problème de calcul. En d'autres termes, nous proposons de mieux maîtriser l'impact du choix des hypothèses d'idéalisation tel que le choix d'un type de matériau (isotrope, orthotrope ...), celui d'un type de loi de comportement (élastique linéaire, bilinéaire, élasto-plastique .. . ), la considération de détails géométriques (congés, perçages, filetages, nervures ... ), la représentation de l'environnement (efforts ponctuels ou répartis, sollicitations simples ou combinées ...) sur la qualité du résultat de la simulation et sur le coût de la simulation.

Pour ceci, l'article se décompose en trois parties. La première décrit les éléments essentiels de la chaîne CAOcalcul dans le processus de conception et les enjeux qui y sont liés. La partie suivante présente plus précisément les éléments essentiels qui aident aux choix des idéalisations des géométries issues de la CAO. La dernière propose une structuration des différentes étapes d'aide aux choix par une méthode qui fait appel à une base de cas. Enfin, un exemple d'application de cette méthode est détaillé dans la section 5 .

\section{Chaîne CAO - Calcul en conception}

En conception, la chaîne CAO-calcul peut être présentée comme une boucle (Fig. 3).

En simulation des systèmes physiques, l'ingénieur de calcul a souvent besoin de modèles géométriques qui doivent représenter le plus fidèlement possible le système réel conçu. Toutefois, des contraintes de coût d'analyse peuvent rendre impossible une simulation de comportement d'une structure modélisée avec tous les détails géométriques. Par conséquent, un ingénieur de calcul doit 


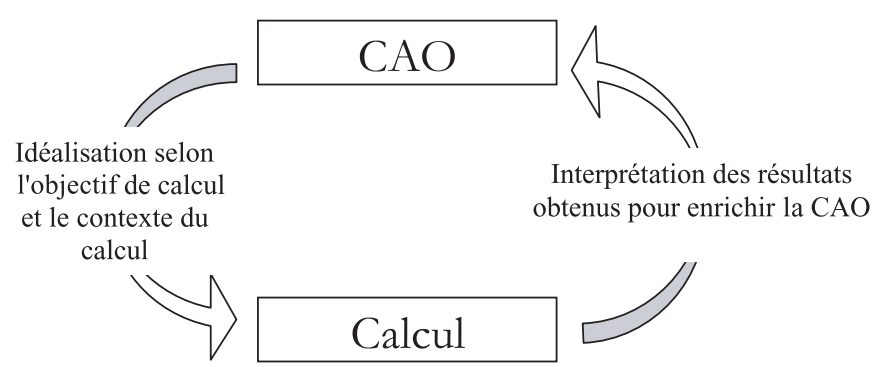

Fig. 3. Représentation de la chaîne CAO - Calcul dans la conception.

connaître le contexte en terme de coût et de qualité pour ce calcul afin qu'il puisse appliquer les idéalisations appropriées. Plusieurs auteurs ont abordé l'intérêt des idéalisations appliquées au calcul. Armstrong et al. dans [9] ont étudié l'influence des idéalisations par suppression de détails, ou par simplifications géométriques sur la précision des résultats de calculs. Des procédures sont développées pour réaliser des simplifications sur la géométrie du modèle à calculer. Shephard et al. dans [10] ont examiné les sources d'idéalisation généralement utilisées dans un calcul. Les sources d'idéalisations sont liées au modèle mathématique, au domaine géométrique, aux propriétés des matériaux, aux conditions aux limites et au chargement. Une approche méthodologique est proposée pour contrôler l'idéalisation mais seules les simplifications géométriques et de chargement sont implémentées.

En conclusion, faire les choix d'idéalisation qui doivent être appliqués sur un modèle issu de la CAO reste jusqu'à nos jours une tâche difficile, réalisée par des experts. Ainsi, notre article présente une méthode d'aide aux choix d'idéalisation en fonction du temps dont on dispose pour le calcul et de la qualité du résultat que l'on attend.

\section{Idéalisation des modèles CAO destinés au calcul}

En conception de produits, le calcul est un outil utilisé dans trois contextes différents [2]. Selon ces contextes, trois types de calcul peuvent être distingués : le calcul de compréhension, le calcul d'aide aux choix et le calcul de validation.

1. Calcul de compréhension : l'objectif ici est d'avoir des résultats de calcul pour aider à comprendre un comportement physique donné sans toutefois avoir besoin de choisir des solutions de conception. Les conclusions des calculs de compréhension peuvent aider aux choix d'idéalisation pour les deux autres types de calcul.

2. Calcul d'aide aux choix : les calculs d'aide aux choix permettent de choisir parmi plusieurs solutions technologiques possibles pour un produit conçu celles qui sont conformes aux besoins de conception. Ces calculs répondent généralement à de fortes contraintes de coût (ou de délai).
3. Calcul de validation : ce type de calcul est utilisé pour la validation des choix. Ils peuvent être contraints à une qualité (précision des résultats fournis par le modèle par rapport au comportement réel) de plus en plus forte au cours du processus de conception.

Pour ces trois contextes d'utilisation du calcul en conception, différents moyens permettent de procéder à l'idéalisation :

1. L'expertise humaine qui peut aider à comparer différentes idéalisations et à choisir la plus adéquate en se basant sur des connaissances d'experts.

2. Les modèles hiérarchiques pour pouvoir classer différents modèles d'idéalisations pour un modèle de conception selon leurs degrés d'adéquation avec le système réel en fonction du niveau de complexité et de représentativité du système réel [11]. L'analyste procède à la comparaison des résultats obtenus pour les différents modèles réalisables par rapport à ceux fournis par la définition la plus complète possible du problème (un domaine géométrique complet, des conditions aux limites peu simplifiées, une loi de comportement de matériau la plus proche possible de la loi réelle, etc.) [12].

3. La quantification des influences sur les résultats des calculs des idéalisations choisies, et étude de la relation entre elles, comme suggéré par Vignjevic et al. dans [13]. Ceci est possible avec l'utilisation des outils statistiques et mathématiques comme la méthode des plans d'expériences utilisée par Benhafid et al. dans $[3,14]$.

Nous jugeons que les points présentés sont des composantes essentielles pour aider à choisir et à comprendre les effets des idéalisations sur les résultats des calculs. La partie suivante présente notre proposition pour formaliser une méthode d'aide à l'idéalisation en calcul de structures.

\section{Méthode d'aide aux choix d'idéalisations pour les modèles $\mathrm{CAO}$}

La méthode d'aide à l'idéalisation proposée se base sur les points suivants :

a. L'hypothèse de base est l'existence de similarité de comportement, et donc de résultats en terme de qualité et coût des modèles, entre différents cas d'analyse. En d'autres termes, nous proposons d'utiliser les résultats de développement obtenus sur un cas d'analyse donné pour aider à la modélisation d'un cas similaire. Le jugement de similarité entre différents cas d'analyse est très couramment utilisé par les analystes. Une analyse, conduite sur un cas d'école (comparaison d'un culbuteur et d'une pièce de poinçonneuse) puis sur un cas industriel (bornes de disjoncteurs présentées en Figs. 7 et 12), de cas jugés a priori similaires par des experts a permis de montrer que pour des cas jugés similaires, l'influence des hypothèses 


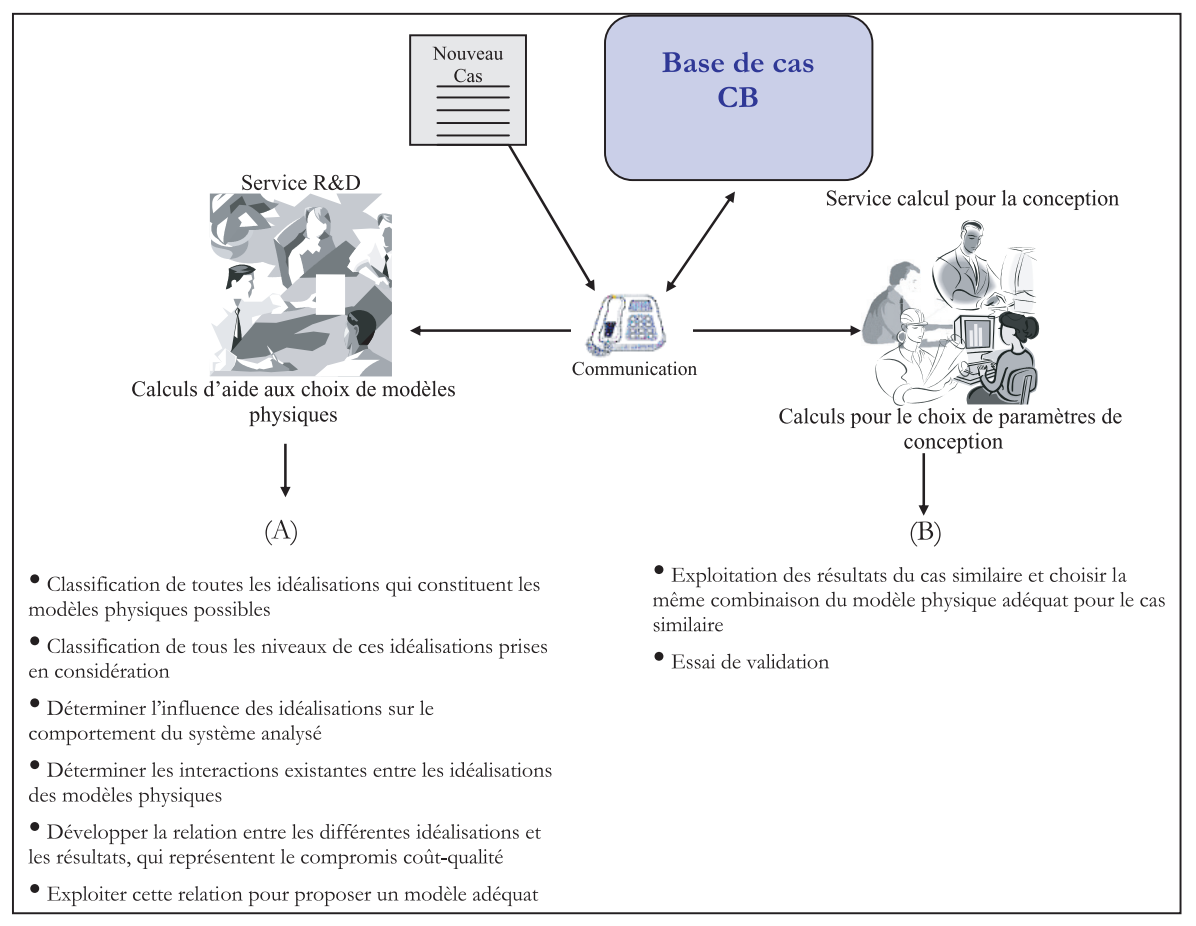

Fig. 4. La méthode d'aide aux choix d'idéalisations.

choisies sur le résultat et les interactions entre les hypothèses sont les mêmes. La figure 5 montre des graphes d'influence obtenus avec la méthode des plans d'expérience pour deux cas analysés jugés a priori similaires. Les graphes des figures $5 \mathrm{a}$ et b ont la même allure sauf que les valeurs des réponses ne sont pas incluses dans les mêmes intervalles. La similarité des graphes correspond à une similarité des comportements des deux cas analysés. Il est alors possible de se fier au jugement des experts pour savoir si un cas de calcul est similaire à un cas de référence. Il est alors possible de se baser sur les résultats obtenus sur le cas de référence pour estimer le comportement du cas à traiter et ainsi choisir l'idéalisation adéquate.

b. Pour les cas les plus fréquents, des calculs sont menés pour construire un cas de référence qui fournit les informations nécessaires pour l'aide à l'idéalisation de cas similaires. La démarche de construction d'un cas de référence est basée sur la méthode des plans d'expérience et est détaillée par Benhafid et al. dans [14]. La méthode des plans d'expérience se base sur l'expression d'un modèle mathématique permettant de formaliser l'influence des hypothèses sur les grandeurs mesurées (temps de calcul, contrainte de Von Mises au point critique, déplacement maximum ... ) et les interactions entre les hypothèses [15].

c. Sur la base de cette formulation mathématique dans le cadre du cas de référence, un traitement automatique permet de fournir l'idéalisation adéquate pour un cas similaire et un contexte de calcul donné (temps disponible pour le calcul, qualité des résultats attendue) en considérant la résolution d'un problème d'optimisation multi-objectif.
La figure 4 présente, de façon globale, la méthode d'aide aux choix d'idéalisation proposée en reprenant les trois points ci-dessus. La mise en relation d'un nouveau cas d'analyse avec la base des cas de référence se fait en utilisant le jugement des experts. Deux démarches peuvent alors être mises en œuvre. La démarche (A) si un cas de référence doit être développé, la démarche (B) si un cas de référence similaire existe et peut être exploité pour choisir les hypothèses nécessaires à l'idéalisation pour un contexte de conception donné. Cette méthode est détaillée dans la section suivante à l'aide d'un exemple d'application.

\section{Exemple d'application}

L'exemple d'application est une borne de disjoncteur présentée dans la figure 6 . Une borne de disjoncteur est une pièce qui sert au raccordement de fils électriques entre le disjoncteur et l'installation électrique. Le fonctionnement de cette borne induit des déplacements, des déformations et des contraintes dans la structure. Pour déterminer les résultats en termes de contrainte de Von Mises et de déplacement dans la borne, un analyste doit effectuer des choix d'idéalisations sur la borne, et réaliser des calculs statiques.

Considérons un but de simulation formulé de la façon suivante : "Estimer les contraintes dans la borne pour préparer une réunion d'avancement du projet lors des étapes amont de conception (aucune solution n'est encore choisie et l'objectif est d'avoir des données comparatives entre différents types de bornes) ». Ce contexte de conception peut se traduire par un coût (ici réduit au temps) de 

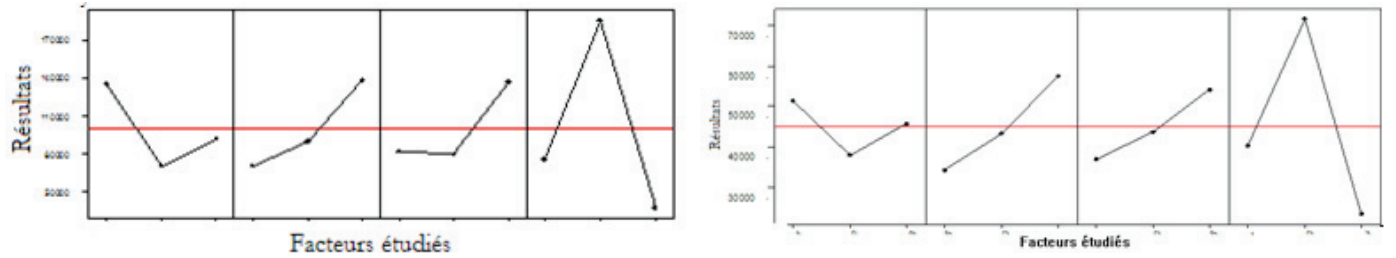

Fig. 5. Deux graphes d'influences pour deux cas différents obtenus par analyse des plans d'expériences.

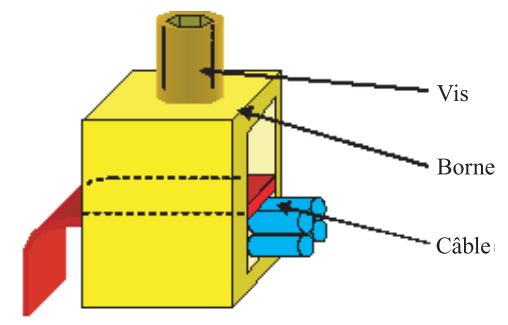

Fig. 6. Description du système borne/vis.

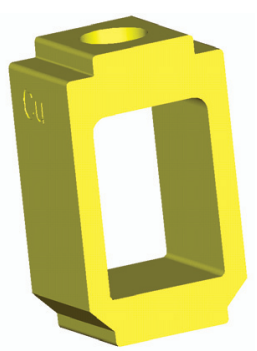

Fig. 7. Borne de disjoncteur.

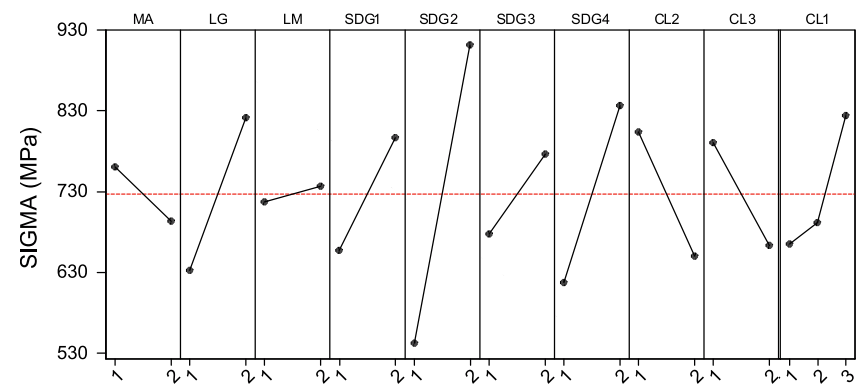

Fig. 8. Graphes d'influences pour les idéalisations pour la borne analysée - Résultats en contraintes.

calcul réduit et un niveau de qualité suffisant pour utiliser une analyse comparative avec d'autres bornes. Cette traduction peut être : « le délai de réponse doit être inférieur à 7 heures » et « la qualité doit être au moins de $75 \%$ ». Un analyste qui applique la méthode donnée dans la figure 4 peut alors procéder de la manière suivante.

Si l'analyse à conduire sur la borne n'est pas jugée similaire à un cas de référence existant mais qu'elle est fréquente, il est possible d'en faire un cas de référence [14]. Une structuration des différentes hypothèses d'idéalisation considérées sur la borne et les niveaux associés est définie dans le tableau 1. La méthode des plans d'expérience est utilisée pour structurer la construction du cas de référence et permet d'obtenir des modèles mathématiques, qui précisent les influences des

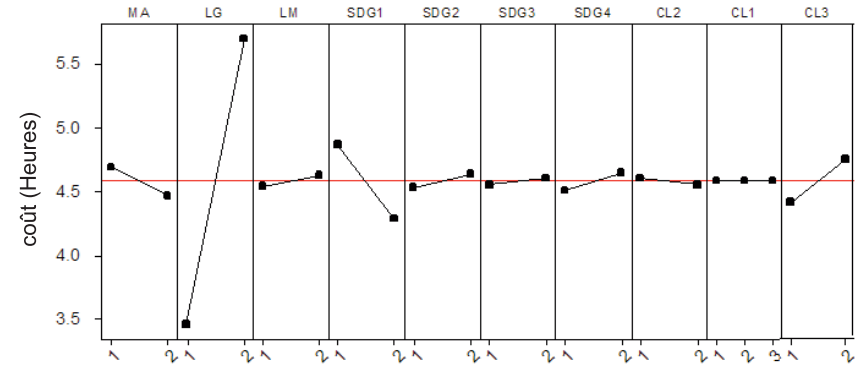

Fig. 9. Graphes d'influences pour les idéalisations pour la borne analysée - Résultats en coût de calcul.

différentes hypothèses sur les résultats et les interactions entre les hypothèses d'idéalisation. Dans le cadre de l'exemple d'application, les modèles mathématiques identifiés sur le cas de référence et concernés par le but de simulation sont pour la contrainte de Von Mises au point critique :

(1) $\sigma(\mathrm{MPa})=727,06+33,28^{*} X_{\mathrm{MA}}-94,72^{*} X_{\mathrm{LM}}$

$-9,56^{*} X_{\mathrm{LG}}-69,61^{*} X_{\mathrm{SDG} 1}-184,72^{*} X_{\mathrm{SDG} 2}-49,5^{*} X_{\mathrm{SDG} 3}$

$-109,22^{*} X_{\mathrm{SDG} 4}-35,22^{*} X_{\mathrm{CL} 1}+77,17^{*} X_{\mathrm{CL} 2}+63,89^{*} X_{\mathrm{CL} 3}$,

et pour le temps de simulation :

(2) Temps (heures) $=4,58333-0,11167^{*} X_{\mathrm{MA}}$

$$
-1,125^{*} X_{\mathrm{LM}}-0,04167^{*} X_{\mathrm{LG}}+0,29167^{*} X_{\mathrm{SDG} 1}
$$

$-0,055^{*} X_{\mathrm{SDG} 2}-0,02833^{*} X_{\mathrm{SDG} 3}-0,07^{*} X_{\mathrm{SDG} 4}+0^{*} X_{\mathrm{CL} 1}$

$$
+0,02833^{*} X_{\mathrm{CL} 2}-0,1667^{*} X_{\mathrm{CL} 3} \text {, }
$$

avec les variables XMA, XML, XLG ... correspondant aux niveaux de simplification des hypothèses d'idéalisation considérées dans le tableau 1 (ces valeurs prennent les valeurs indiquées dans Tab. 1).

Différents modes de visualisation peuvent être construits à partir de l'identification de ces relations (graphes d'influence et d'interaction présentés en Figs. 810), mais la formalisation mathématique fournie par (1) et (2) permettra de fournir automatiquement et très rapidement le modèle à choisir pour un cas similaire.

Si l'analyse à conduire est jugée similaire à un cas de référence existant (par exemple le cas de référence est construit sur la borne de la Fig. 7 et la simulation à réaliser concerne celle de la Fig. 12), les relations mathématiques du cas de référence (telles que (1) et (2)) sont utilisées pour générer rapidement toutes les réponses possibles en délai et contraintes de Von Mises au point critique. Le problème peut alors être formulé sous la forme 
Tableau 1. Description des sigles des idéalisations pour les modèles physiques.

\begin{tabular}{|c|c|c|c|}
\hline Idéalisation & $\begin{array}{c}\text { Niveau } \\
\text { de simplification }\end{array}$ & Description du niveau & \\
\hline Type de matériau & $\begin{array}{l}1 \\
2\end{array}$ & $\begin{array}{l}\text { Matériau isotrope } \\
\text { Matériau anisotrope }\end{array}$ & $\mathrm{MA}$ \\
\hline $\begin{array}{l}\text { Simplification de détail } \\
\text { géométrique : rayons }\end{array}$ & $\begin{array}{l}1 \\
2\end{array}$ & $\begin{array}{l}\text { Oter les rayons } \\
\text { Prendre en considération les rayons }\end{array}$ & SDG1 \\
\hline $\begin{array}{l}\text { Simplification de détail } \\
\text { géométrique : trou de sertissage }\end{array}$ & $\begin{array}{l}1 \\
2\end{array}$ & $\begin{array}{l}\text { Ôter le trou de sertissage } \\
\text { Prendre en considération le trou de sertissage }\end{array}$ & SDG2 \\
\hline $\begin{array}{l}\text { Simplification de détail } \\
\text { géométrique : forme du haut }\end{array}$ & $\begin{array}{l}1 \\
2\end{array}$ & $\begin{array}{l}\text { Forme simple du haut de la borne } \\
\text { Forme réelle du haut de la borne }\end{array}$ & SDG3 \\
\hline $\begin{array}{l}\text { Simplification de détail } \\
\text { géométrique : forme du bas }\end{array}$ & $\begin{array}{l}1 \\
2 \\
\end{array}$ & $\begin{array}{l}\text { Forme simple du bas de la borne } \\
\text { Forme réelle du bas de la borne }\end{array}$ & SDG4 \\
\hline $\begin{array}{l}\text { Condition à la limite } 1: \\
\text { traction de la borne }\end{array}$ & $\begin{array}{l}1 \\
2 \\
3\end{array}$ & $\begin{array}{l}\text { Chargement ponctuel (traction) } \\
\text { Chargement surfacique } \\
\text { Chargement surfacique }+ \text { couple de torsion }\end{array}$ & CL1 \\
\hline $\begin{array}{l}\text { Condition à la limite } 2: \\
\text { maintien de la borne }\end{array}$ & $\begin{array}{l}1 \\
2\end{array}$ & $\begin{array}{l}\text { Maintien ponctuel } \\
\text { Maintien surfacique }\end{array}$ & CL2 \\
\hline $\begin{array}{l}\text { Condition à la limite } 3: \\
\text { Câbles-borne }\end{array}$ & $\begin{array}{l}1 \\
2\end{array}$ & $\begin{array}{l}\text { Contact ponctuel } \\
\text { Pression surfacique }\end{array}$ & CL3 \\
\hline Linéarité géométrique & $\begin{array}{l}1 \\
2\end{array}$ & $\begin{array}{l}\text { Linéarité géométrique } \\
\text { Non-linéarité géométrique }\end{array}$ & $\overline{L G}$ \\
\hline Linéarité matérielle & $\begin{array}{l}1 \\
2\end{array}$ & $\begin{array}{l}\text { Linéarité matérielle } \\
\text { Non-linéarité matérielle }\end{array}$ & LM \\
\hline
\end{tabular}

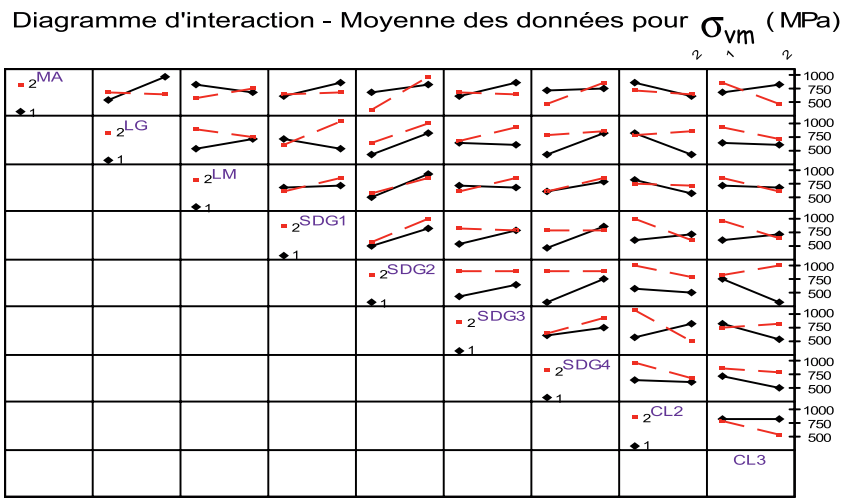

Fig. 10. Diagrammes d'interactions entre les hypothèses de modèles pour la borne analysée - Résultats en contraintes de Von Mises.

d'un problème d'optimisation multi-objectifs : parmi l'ensemble des modèles possibles, quel est celui qui minimise à la fois l'écart entre la qualité voulue et la qualité obtenue et celui entre le délai accordé et le temps de calcul?

Pour répondre à ce problème, une fonction de désirabilité [16] est construite pour combiner les deux objectifs qui sont d'obtenir un délai inférieur à 7 heures et d'obtenir une qualité minimum de $75 \%$. La définition d'une fonction de désirabilité est associée à chaque fonction objectif comme le préconise [17]. Soient $d_{\text {réponse } 1}$ la contrainte de Von Mises obtenue au point critique et $d_{\text {réponse } 2}$ le temps de simulation. La désirabilité globale $d$, est définie par le produit : $d=\sqrt{d_{\text {réponse } 1} * d_{\text {réponse } 2}}$.

Il est à noter que, pour le moment, nous avons défini la qualité, $Q$, comme le rapport entre le résultat fourni par

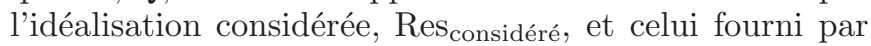

Diagramme d'interaction - Moyenne des données pour coût (Heures)

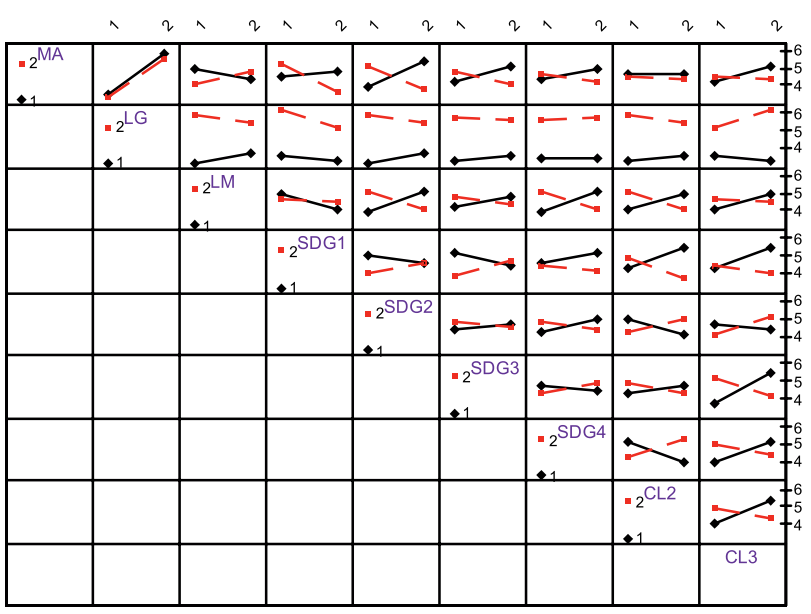

Fig. 11. Diagrammes d'interactions entre les hypothèses de modèles pour la borne analysée - Résultats en coût de calculs.

l'idéalisation mettant en ouvre le modèle le plus complet,

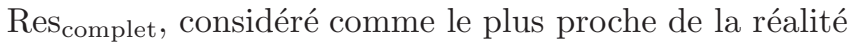

$$
\text { (3) } Q=\text { Res }_{\text {considéré}} / \operatorname{Res}_{\text {complet }}
$$

Le modèle donnant la plus grande désirabilité est alors considéré comme étant celui à mettre en œuvre pour l'analyse à réaliser. Dans le cadre de l'exemple d'application, le maximum est recherché parmi les 1536 valeurs de $d$ possibles. Le modèle idéalisé à mettre en œuvre est alors celui qui correspond au maximum de $d$. En d'autres termes, c'est le modèle qui répond le mieux aux objectifs de coût de calcul et de qualité de résultats pour les contraintes de Von Mises et le coût de calcul. 


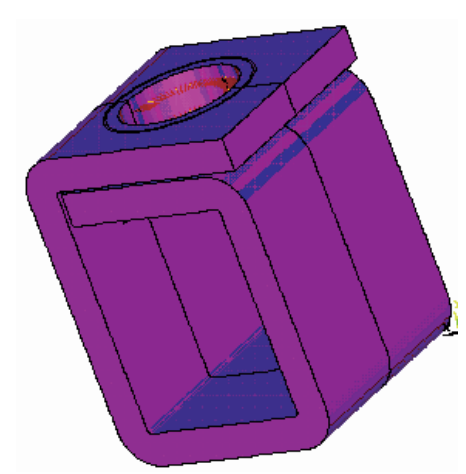

Fig. 12. Borne similaire.

Dans le cas de la borne, le modèle idéalisé maximisant la désirabilité à réaliser correspond au choix des hypothèses suivantes : matériau isotrope ( $\mathrm{MA}=1$ dans Tab. 1), petits déplacements, petites déformations ( $\mathrm{LG}=$ 1 dans Tab. 1), loi de comportement linéaire ( $\mathrm{LM}=1$ dans Tab. 1), prise à compte des rayons de raccordement (SDG1 = 2 dans Tab. 1), ne pas considérer le trou de sertissage (SDG2 = 1 dans Tab. 1), simplification de la géométrie du haut de la borne (SDG3 = 1 dans Tab. 1), simplification de la géométrie du bas de la borne (SDG4 = 1 dans Tab. 1), effort ponctuel appliqué en haut de la borne (CL1 = 1 dans Tab. 1$)$, maintien surfacique sur les côtés de la borne (CL2 = 2 dans Tab. 1 ), effort ponctuel appliqué en bas de la borne (CL3 = 1 dans Tab. 1). Ce modèle correspond à une qualité prévue de $Q=77 \%$, et un temps de calcul de $T=6,50$ heures. Ce modèle est celui qui respecte le mieux le compromis coût/qualité spécifié.

\section{Conclusion}

Notre article propose une méthode d'aide aux choix d'idéalisation de calcul dans la conception de produits. La méthode proposée s'appuie sur la similarité entre les cas d'analyse : cette propriété est utilisée généralement par des experts d'entreprises ou de bureaux de recherche et développement. La construction et la structuration de cas de référence aide un analyste expert comme un non expert à conduire l'idéalisation pour un cas similaire. Les cas de référence sont construits à l'aide de la méthode des plans d'expérience qui repose sur la mise en place d'un modèle mathématique. Ce modèle permet de repérer automatiquement les facteurs les plus influents ainsi que les niveaux de dépendance entre les facteurs. En d'autres termes, en utilisant le modèle mathématique fourni par les plans d'expérience sur le cas de référence, pour un contexte de conception donnée (traduit sous la forme d'un niveau de qualité et d'un coût objectif pour l'analyse), un traitement automatique est proposé, qui donnent les hypothèses d'idéalisation à choisir pour un cas similaire au cas de référence.

Remerciements. Nous remercions Schneider Electric pour les données fournies dans le cadre de cette étude.

\section{Références}

[1] B.A. Szabo, The problem of model selection in numerical simulation, 3rd international conference of computational structures technology, Advances in computational methods for simulation, Budapest, 1996

[2] N. Troussier, Contribution à l'intégration du calcul mécanique dans la conception de produits techniques : proposition méthodologique pour l'utilisation et la réutilisation, Thèse Université Joseph Fourier, Grenoble, 1999

[3] Y. Benhafid, N. Troussier, N. Boudaoud, Towards the use of the design of experiments method to control the quality of structural analysis, Proceedings of the 8th international conferences on Quality, Reliability, Maintainability, CCF, Sinaia (Roumania) : Mediarex 21, 2002

[4] M. Breuer, Numerical and modeling influences on large eddy simulations for the flow past a circular cylinder, Int. J. Heat and Fluid Flow 19(5) (1998) 512-521

[5] P. Kurowski, B. Szabo, How to find errors in finiteelement models, Machine design (1997) 93-98

[6] A. Umar et al., Prediction of error in finite element results, Computers \& Structures 60(3) (1996) 471-480

[7] R. Vignjevic, A.J. Morris, A.D. Belagundu, Towards high fidelity finite element analysis, Advances in Engineering Software 29(7-9) (1998) 655-665

[8] F. Pourroy, Apport des éléments finis à la conception mécanique, Les Techniques de l'Ingénieur, Réf. AG2530.

[9] C.G. Armstrong, R.J. Donaghy, S.J. Bridgett, Derivation of appropriate idealisations in finite element modelling, Advances in Finite Element Technology, Civil-Comp Press, 1996, pp. 11-20

[10] M.S. Shepard, P.L. Baehmann, M.K. Georges, E.V. Korngold, Framework for the reliable generation and control of analysis idealization, Computer methods in applied mechanics and engineering 82 (1990) 257-280

[11] B. Szabó, I. Babuška, Mathematical models and engineering decisions, Finite Element Analysis, J.W. Sons, Editor, 1991, pp. 1-9

[12] M.S. Shepard, E.V. Korngold, R.R. Collar, P.L. Baehmann, A modeling framework for controlling structural idealizations in engineering design, Computers and Structures 37(2) (1990) 181-191

[13] R. Vignjevic, A.J. Morris, A.D. Belegundu, Reliable finite element analysis through hierarchical decomposition of the idealisation process, Proceedings of the 6th International Conference on Civil and Structural Engineering Computing, 1995

[14] Y. Benhafid, N. Troussier, N. Boudaoud, Z. Cherfi, Towards a modelling assistance method in structural analysis, Application to a terminal system of circuit breaker, 5th International Conference on Integrated Design and Manufacturing in Mechanical Engineering (IDMME'04), Bath, UK, 2004

[15] P. Schimmerling, J.C. Sisson, A. Zaïdi, Pratique des plans d'expériences, Lavoisier TEC et DOC, Paris, 1998

[16] E. Harrington, Experiments; Statistical process control (SPC); Industrial Quality Control 21(10) (1965) 494-498

[17] E. Del Castillio, C.D. Montgomery, D.R. McCarville, Modified Desirability Fonctions for Multiple Response Optimization, J. Quality Technology 28(3) (1996) 337-345 